\section{Mutations lurk in regulatory regions}

Cancer-genome sequencing has yielded a long list of potential cancer-causing mutations, most of which are in genes that code for proteins. But two studies of melanoma genomes have revealed common mutations in a region that regulates gene expression.

Dirk Schadendorf of the University Hospital Essen in Germany, Rajiv Kumar of the German Cancer Research Center in Heidelberg and their colleagues conducted a genetic analysis of 14 members of a family that is prone to the skin cancer. The authors found mutations in a region that regulates the expression of a gene called TERT. Another group led by Levi Garraway at the Dana-Farber Cancer Institute in Boston, Massachusetts, found mutations in the same promoter region in 50 out of 70 melanomas. The results suggest that regulatory regions of the genome may be key reservoirs of cancer-causing mutations. Science http://dx.doi. org/10.1126/science.1230062; http://dx.doi.org/10.1126/ science.1229259 (2013)

\section{NEUROSCIENCE}

\section{Old age, bad sleep, poor memory}

The gradual loss of cells in the brain's cortex could be decreasing sleep quality in older adults, leading to poorer long-term memory.

Bryce Mander and Matthew Walker at the University of California, Berkeley, and their group asked healthy adults to memorize a list of words, recall some of them ten minutes later, and recall the rest the next morning. Adults in their late 60 s and their 70 s performed worse on the test, and showed significant reductions in the slow brain waves that are associated with deep sleep, compared with those around the age of 20 . The extent of deep-sleep disruption was related to the degree of memory impairment, and these differences were, in turn, linked with a reduction of grey matter in the medial prefrontal cortex.

The findings suggest that deterioration of this part of the brain diminishes the slow brain waves, which are implicated in memory consolidation, impairing the ability to solidify new memories.

Nature Neurosci. http://dx.doi. org/10.1038/nn.3324 (2013)

\section{GENE THERAPY}

\section{Gene fix does not prevent cell loss}

Gene therapy improves the vision of people with a genetic form of blindness, but does not stop the loss of the lightsensitive photoreceptor cells in the retina.

Childhood blindness as a result of Leber congenital amaurosis (LCA) occurs as a result of photoreceptor dysfunction and degeneration, owing to a mutation in the gene $R P E 65$. In addition to fixing the dysfunction, researchers hoped therapy with a working copy of the gene would slow the loss of the cells. But, Artur Cideciyan at the University of Pennsylvania in Philadelphia and his group found that the loss continues. In dogs bearing the LCA mutation, those that were treated at a disease stage approximating to that in humans also showed photoreceptor deterioration.

The results suggest that treatment for this hereditary blindness also needs to address long-term protection of the light-sensing cells.

Proc. Natl Acad. Sci. USA http://dx.doi.org/10.1073/ pnas.1218933110 (2013)

$$
\text { ZOOLOGY }
$$

\section{Turtle arrested development}

Some reptile species give birth to live young, but turtles have never evolved to do so - perhaps because of low oxygen levels in their

COMMUNITY CHOICE

The most viewed papers in science

\title{
GENOMICS
}

\section{Disease genes mutate more}

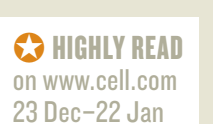

Genetic mutations occur at random, but where in the genome they occur is nonrandom. Jonathan Sebat at the University of California, San Diego, Jun Wang at

BGI-Shenzhen in China and their group report that some regions of the genome mutate a 100 times more frequently than others, and that genes linked to autism have higher than average mutation rates.

To track emerging mutations, the researchers sequenced the complete genomes of ten sets of identical twins with autism spectrum disorder and their parents. The analysis revealed 'hotspots' where new mutations tend to cluster and showed that mutation rates were associated with certain DNA sequences or with specific aspects of how the DNA is packaged. Disease genes - including those implicated in autism - showed high mutation rates, as did those expressed in the brain.

Further study of mutation hotspots could help researchers to identify more genetic risk variants, and to better understand human variability and genome evolution.

Cell 151, 1431-1442 (2012)

egg-laying tubes, or oviducts.

Anthony Rafferty at

Monash University in Clayton, Australia, and his group show that oxygen diffused more slowly in secretions from the oviducts of four species of turtle than in saline solution. When the turtles' eggs were incubated at low oxygen levels they stopped developing, whereas those kept at ambient conditions developed normally. The low oxygen levels in the oviducts could explain how turtles are able to store eggs in a state of arrested development until they can lay them on land.

Am. Nat. http://dx.doi. org/10.1086/668827 (2013)

\section{BIOTECHNIQUES}

\section{Cell squeezer gets molecules in}

The cell membrane, largely impermeable to large molecules, can be breached with needles, electricity and chemicals. But now researchers have devised a less traumatic and more efficient way of delivering molecules into cells,

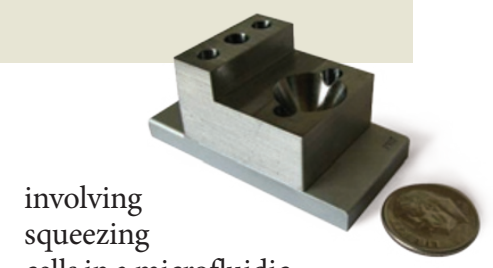

cells in a microfluidic

device (pictured).

A team led by Klavs Jensen and Robert Langer at the Massachusetts Institute of Technology in Cambridge found that passing various cells through the micrometrewide channels of their device deforms the cells, creating temporary holes in the cell membrane that allow large molecules to pass through. The new approach is 10 to 100 times more efficient than conventional methods at delivering proteins into human skin cells to reprogram them into stem-cell-like cells.

The technique could be used to deliver therapeutic molecules into human cells, the authors say.

Proc. Natl Acad. Sci. USA http://dx.doi.org/10.1073/ pnas.1218705110 (2013)

\section{NATURE.COM}

For the latest research published by Naturevisit:

www.nature.com/latestresearch 